\title{
EFFECTIVE INVARIANT THEORY OF PERMUTATION GROUPS USING REPRESENTATION THEORY
}

\author{
NICOLAS BORIE
}

\begin{abstract}
Using the theory of representations of the symmetric group, we propose an algorithm to compute the invariant ring of a permutation group. Our approach have the goal to reduce the amount of linear algebra computations and exploit a thinner combinatorial description of the invariant ring.

Computational Invariant Theory, representation theory, permutation group

This is a drafty old version : full corrected text are available at : http://www.springer.com/
\end{abstract}

\section{INTRODUCTION}

Invariant theory has been a rich and central area of algebra ever since the eighteenth theory, with practical applications [6, § 5] in the resolution of polynomial systems with symmetries (see e.g. [4], [10, [20, § 2.6], [7]), in effective Galois theory (see e.g. 5], 1], [11]), or in discrete mathematics (see e.g. [22, 16] for the original motivation of the second author). The literature contains deep and explicit results for special classes of groups, like complex reflection groups or the classical reductive groups, as well as general results applicable to any group. Given the level of generality, one cannot hope for such results to be simultaneously explicit and tight in general. Thus the subject was effective early on: given a group, one wants to calculate the properties of its invariant ring. Under the impulsion of modern computer algebra, computational methods, and their implementations, have largely expanded in the last twenty years $[12,20,23,6,13,14$. However much progress is still needed to go beyond toy examples and enlarge the spectrum of applications.

Classical approaches solving the problem of computing invariant ring use elimination techniques in vector spaces of too high dimensions. Gröbner basis become impracticable when the number of variables goes up (around 10 for modern computers). The evaluation approach proposed by the author in [3] required a permutation group whose index in the symmetric group is relatively controlled (around 1000 for modern computers). Each approaches localize the algebra reduction in vector spaces still of too large dimensions. Gröbner basis approaches works with monomials of degree $d$ over $n$ variables and linear reduction over a space spanned by these monomials is costly. The evaluation approach, proposed by the author in his thesis, does the linear algebra in a free module spanned by the cosets of the symmetric group by a permutation group (i.e. it have the index for dimension). In both case, as linear reduction globally cost the cube of the dimension of the space, one cannot hope to go so much further with classical approaches even with the progress of computer.

We propose in this article an approach following the idea that adding more combinatorics in invariant theory help to produce more efficient algorithms whose outputs could perhaps reveal some combinatorics also ; the long time goal being 
having a combinatorial description of invariant ring (generators or couple of primary secondary invariants families). Since Hilbert, This problem have been solve only in very restrictive and special cases (for example, 9] give secondary invariants for Young subgroups of symmetric groups). We focus on the problem of computing secondary invariants of finite permutation groups in the non modular case. Assuming that, we will shows how to localize computations inside selected irreducible representations of the symmetric group. These spaces are smaller than the ones used in classical approaches and we can largely take advantages of the combinatorial results coming from the theory of representations of the symmetric group.

\section{INVARIANT RING AND REPRESENTATIONS OF THE SYMMETRIC GROUP}

For any set $S$, we will denote $|S|$ the cardinality of the set $S$.

2.1. Invariant ring of permutation group and application to combinatorics. Our approach start from a result in one key article of invariant theory written by Stanley [18, proposition 4.9] mixing invariant of finite group and combinatorics. We recall this general result:

Proposition 2.1. Let $\theta_{1}, \ldots, \theta_{n}$ be an homogeneous set of parameters for $\mathbb{K}[\mathbf{x}]^{G}$, where $G$ is any finite subgroup of $G L\left(\mathbb{K}^{n}\right)$ of order $|G|$. Set $d_{i}=\operatorname{deg}\left(\theta_{i}\right)$ and $t=d_{1} \ldots d_{n} /|G|$. Then the action of $G$ on the quotient ring $S=\mathbb{K}[\mathbf{x}] /\left(\theta_{1}, \ldots, \theta_{n}\right)$ is isomorphic to $t$ times the regular representation of $G$.

Applying this result to $\mathfrak{S}_{n}$ the symmetric group of degree $n$ with $\theta_{i}=e_{i}$ (elementary symmetric polynomial)

$$
e_{i}:=\sum_{P \subset\{1, \ldots, n\}}\left(\prod_{i \in P} x_{i}\right),
$$

we recover the well known result that the ring $\mathbb{K}[\mathbf{x}] /\left(e_{1}, \ldots, e_{n}\right)$ is isomorphic the regular representation $R R\left(\mathfrak{S}_{n}\right)$ of the symmetric group (here $t=\frac{n !}{n !}=1$ ). This well known quotient $\mathbb{K}[\mathbf{x}] /\left(e_{1}, \ldots, e_{n}\right)$ is called the coinvariant ring of the symmetric group in the algebraic combinatorics world and several basis of this ring have been explicitly built (Harmonic polynomials, Schubert Polynomials, Descents monomials and more [2, 15, 9]).

$$
\mathbb{K}[\mathbf{x}] /\left(e_{1}, \ldots, e_{n}\right) \sim_{\mathfrak{S}_{n}} R R\left(\mathfrak{S}_{n}\right)
$$

Let $G$ a group of permutations, subgroup of $\mathfrak{S}_{n}$, we know reapply the result of Stanley to $G$ with the same homogeneous set of parameters formed with the elementary symmetric polynomials. Then, the ring of coinvariant of the symmetric group is now also isomorphic to $t=n ! /|G|$ time the regular representation of the group $G$.

$$
\mathbb{K}[\mathbf{x}] /\left(e_{1}, \ldots, e_{n}\right) \sim_{G} \bigoplus_{i=1}^{n ! /|G|} R R(G)
$$

We know that for any permutation group $G$, in the non modular case, the ring of invariant under the action of $G$ is a Cohen-Macaulay algebra. This imply that there exist a family of generator $\eta_{i}$ making the ring of invariant under the action of $G$ a free module of rank $n ! /|G|$ over the ring of symmetric polynomials. 


$$
\mathbb{K}[\mathbf{x}]^{G}=\bigoplus_{i=1}^{n ! / G \mid} \eta_{i} \mathbb{K}\left[e_{1}, \ldots, e_{n}\right]
$$

Taking the quotient on both side by the ideal $\left(e_{1}, \ldots, e_{n}\right)$ and keeping $\eta_{i}$ as representative of its equivalent class in the quotient, we have

$$
\mathbb{K}[\mathbf{x}]^{G} /\left(e_{1}, \ldots, e_{n}\right)=\bigoplus_{i=1}^{n ! /|G|} \mathbb{K} \cdot \eta_{i}
$$

As $\mathbb{K}[\mathbf{x}]^{G}$ is, by definition, the subspace of $\mathbb{K}[\mathbf{x}]$ on which the action of $G$ is trivial, the result of Stanley imply in particular that the polynomials $\eta_{i}$ span the subspace of the coinvariant of the symmetric group on which the action of $G$ is trivial. A way to construct the $\eta_{i}$ is thus to search them as $G$-fixed point inside the ring of coinvariant of the symmetric group and that could be done irreducible representation by irreducible representation.

The theory of representations of the symmetric group has been largely studied, this bring us to formulate the following problem:

Problem 2.2. Let $n$ a positive integer and $G$ a permutation group, subgroup of $\mathfrak{S}_{n}$. Construct an explicit basis of the trivial representations of $G$ appearing in each irreducible subrepresentation of $\mathfrak{S}_{n}$ inside the quotient $\mathbb{K}[\mathbf{x}] /\left(e_{1}, \ldots, e_{n}\right)$.

A first step to solve this problem constitute in having a basis of the coinvariant for the symmetric group respecting the action of $\mathfrak{S}_{n}$ such that it can be partitioned by irreducible representations. We expose this basis in the next section.

2.2. Representations of the symmetric group. We recall in this section some results describing the irreducible representations of the symmetric group.

For a positive integer $n$, we will call $\lambda$ partition of $n$ (denoted $\lambda \vdash n$ ) a non increasing sequence of integers $\lambda=\left(\lambda_{1}, \ldots, \lambda_{r}\right)$ whose entries sum up to $n$.

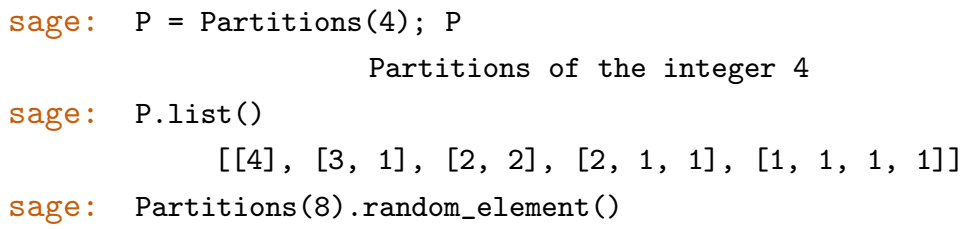

For a positive integer $n$, irreducible representations of the symmetric group $\mathfrak{S}_{n}$ are indexed by the Partitions of $n$. Since we have a finite group, the multiplicity of an irreducible representation inside the regular representation equal its dimension. This information can be collected studying standard tableaux.

Let $n$ a positive integer and $\lambda=\left(\lambda_{1}, \ldots, \lambda_{r}\right)$ a partition of $n$. A tableau of shape $\lambda$ is a diagram of square boxes disposed in raw such that the first raw contains $\lambda_{1}$ boxes, on top of its, a second raw contains $\lambda_{2}$ boxes and so on. A standard tableau of shape $\lambda$ is a filled tableau of shape $\lambda$ with integer from 1 to $n$ such that integers are increasing in column and raw. We will denote $\operatorname{STab}(\lambda)$ the set of standard tableaux of shape $\lambda$.

We can ask Sage to display a such object. 


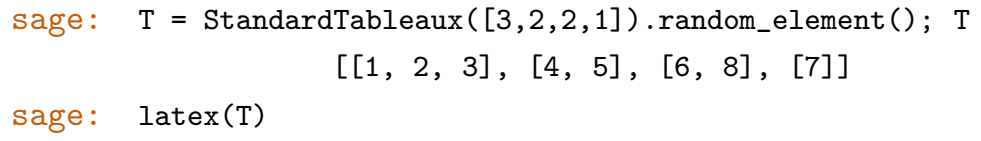

We can also iterate or generate all tableaux of a given shape.

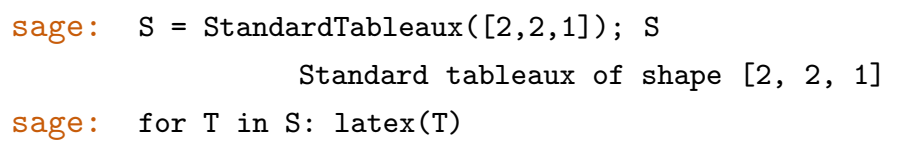

The number $f^{\lambda}$ of standard tableaux of a given shape $\lambda$ can be easily computed using the hook-length formula [2, formula 2.1]. As standard tableaux of shape $\lambda$ constitute a basis indexing of the vector space associated to the irreducible representation of the symmetric group indexed by $\lambda$, and because this same representation must have for multiplicity its dimension inside the regular representation of $\mathfrak{S}_{n}$, we have

$$
\sum_{\lambda \vdash n}\left(f^{\lambda}\right)^{2}=\left|\mathfrak{S}_{n}\right|=n !
$$

The following computation illustrate this equality and check that hook-length formula is well implemented in Sage.

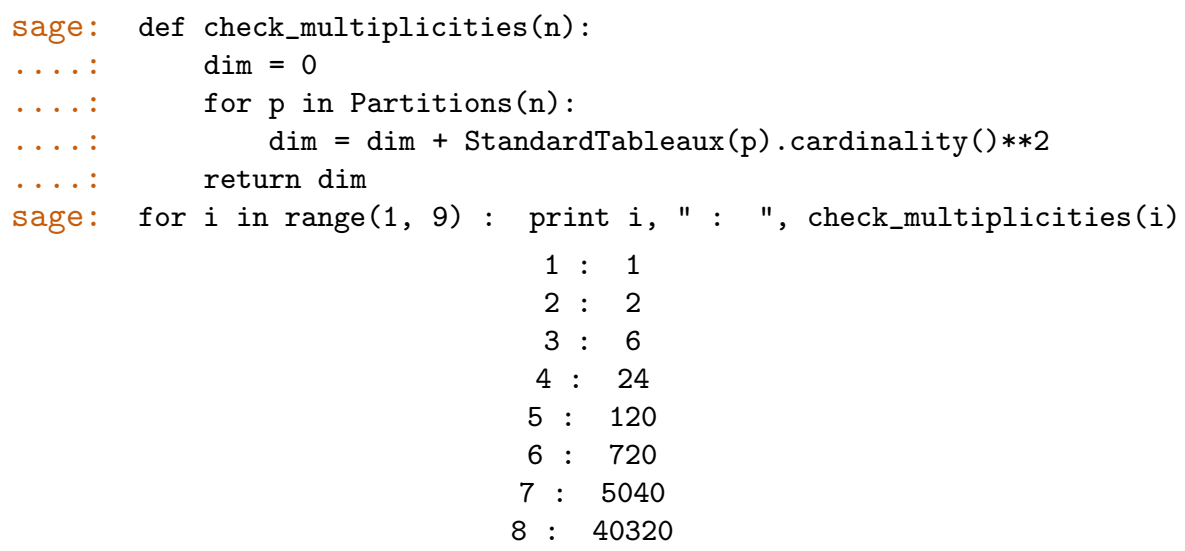

We know describe a last useful object for our algorithmic to come which gather information about all irreducible representations of the symmetric group : the character table.

We recall that character of a representation is a map which associate the trace of matrices for each group element. Such map are constant on conjugacy classes and conjugacy classes of the symmetric group are indexed also by partitions. Any permutation $\tau \in \mathfrak{S}_{n}$ have a single disjoint cycles representation and it belong to 
the conjugacy class indexed by the partition $\mu=\left(\mu_{1}, \ldots, \mu_{r}\right)$ if its disjoint cycles representation contains $r$ cycles of size respectively $\mu_{1}, \mu_{2}, \ldots, \mu_{r}$.

The character table of the symmetric group gather in a square matrices the value of characters of irreducible representations on each conjugacy classes of the symmetric group.

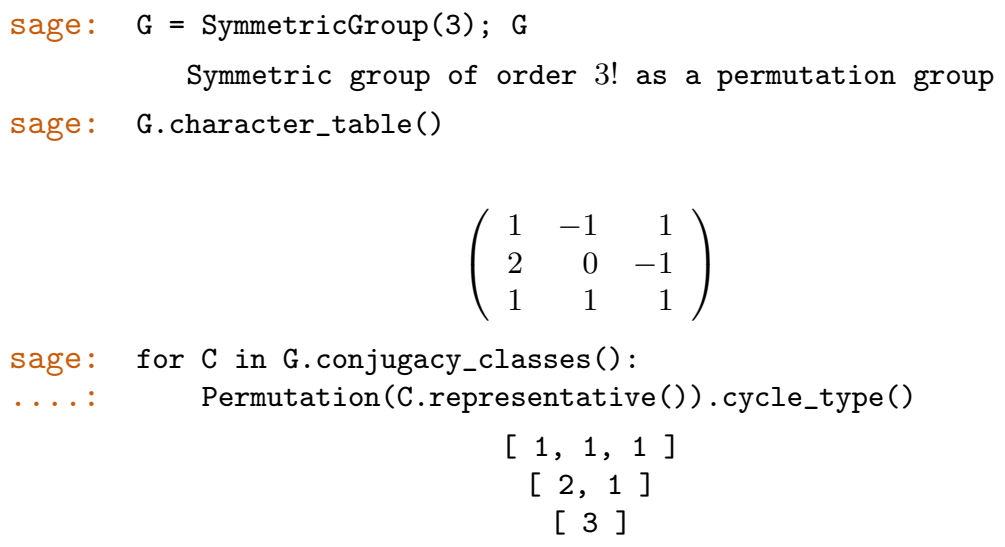

\section{Higher Specht Polynomials for the Symmetric Group}

Algorithmic in invariant theory must, at some point, construct invariant polynomials. Most current approaches use the Reynolds operator or an orbit sum over a group of a special monomial. When the group become large, such invariant become very large even they are stored in a sparse manner inside a computer, the number of terms can easily fit with $n$ ! when $G$ is a permutation group with a small index in $\mathfrak{S}_{n}$.

For our approach, with focuses on the combinatorics of the quotient $\mathbb{K}[\mathbf{x}] /\left(e_{1}, \ldots, e_{n}\right)$, the higher specht polynomials will constitute the perfect family to get explicit answer to Problem 2.2 .

The quotient $\mathbb{K}[\mathbf{x}] /\left(e_{1}, \ldots, e_{n}\right)$ is isomorphic to the regular representation of $\mathfrak{S}_{n}$ in which we have several copies of irreducible representation following their dimension. The Specht polynomials, which are associated standard tableaux, allows to construct explicit subspace of $\mathbb{K}[\mathbf{x}]$ isomorphic to an irreducible representation of the symmetric group. For $\lambda \vdash n$ a partition, the span of Specht polynomials associated to standard tableaux of shape $\lambda$ realize explicitly the irreducible representation of $\mathfrak{S}_{n}$ indexed by $\lambda$. Now, we will see that the higher Specht polynomial take care of multiplicities of irreducible representation inside the coinvariant. They are indexed by pair of standard tableaux of the same shape and they constitute a basis of the $\mathfrak{S}_{n}$-module $\mathbb{K}[\mathbf{x}] /\left(e_{1}, \ldots, e_{n}\right)$. Among all known basis of the coinvariants for the symmetric group (Harmonic, Schubert, monomials under the staircase, descents monomials, ...), the higher Specht polynomial constitute a basis which can be partitioned by irreducible $\mathfrak{S}_{n}$-module by construction.

let $\lambda \vdash n$ a partition and $S, T$ two standard tableaux of shape $\lambda$. We define the word $w(S)$ by reading the tableau $S$ from the top to the bottom in consecutive columns, starting from the left. The number 1 in the word $w(S)$ as for index 0 . Now, recursively, if the number $k$ in the word has index $p$, then $k+1$ has index $p+1$ if it lies to the left of $k$ in the word, it has index $p$ otherwise. For example, 
with the two tableaux

$$
S=\begin{array}{l|l|l}
3 & 5 & \\
\hline 1 & 2 & 4
\end{array} \quad T=\begin{array}{|l|l|l|}
2 & 4 & \\
\hline 1 & 3 & 5 \\
\hline
\end{array}
$$

The reading of the Tableau $S$ give 31524, now placing step by step the indices, we get

$$
\begin{array}{rcc}
31_{0} 524 & \text { initialization } \\
31_{0} 52_{0} 4 & \text { right }: 0 \rightarrow 0 \\
& 3_{1} 1_{0} 52_{0} 4 & \text { left }: 0 \rightarrow 1 \\
& 3_{1} 1_{0} 52_{0} 4_{1} & \text { right }: 1 \rightarrow 1 \\
w(S)= & 3_{1} 1_{0} 5_{2} 2_{0} 4_{1} & \text { left }: 1 \rightarrow 2
\end{array}
$$

Filling the index in corresponding cell of the tableau $S$, We obtain $i(S)$, the index tableau of $S$.

\begin{tabular}{|c|c|c|c|}
\hline$S$ & $T$ & $i(S)$ & $\mathbf{x}_{T}^{i(S)}$ \\
\hline \begin{tabular}{|l|l|l|}
1 & 2 & 3 \\
\end{tabular} & \begin{tabular}{|l|l|l|}
1 & 2 & 3 \\
\end{tabular} & \begin{tabular}{|l|l|l|}
0 & 0 & 0 \\
\end{tabular} & 1 \\
\hline \begin{tabular}{|l|}
3 \\
\end{tabular} & 3 & 1 & $x_{3}$ \\
\hline \begin{tabular}{|l|l|}
1 & 2 \\
\end{tabular} & \begin{tabular}{|l|l|}
1 & 2 \\
\end{tabular} & \begin{tabular}{|l|l|}
0 & 0 \\
\end{tabular} & \\
\hline \begin{tabular}{|l|}
3 \\
\end{tabular} & 2 & 1 & $x_{2}$ \\
\hline \begin{tabular}{|l|l|}
1 & 2 \\
\end{tabular} & \begin{tabular}{|l|l|}
1 & 3 \\
\end{tabular} & \begin{tabular}{|l|l|}
0 & 0 \\
\end{tabular} & \\
\hline \begin{tabular}{|l|}
2 \\
\end{tabular} & 3 & 1 & $x_{2} x_{3}$ \\
\hline \begin{tabular}{|l|l|}
1 & 3 \\
\end{tabular} & \begin{tabular}{|l|l|}
1 & 2 \\
\end{tabular} & \begin{tabular}{|l|l|}
0 & 1 \\
\end{tabular} & \\
\hline 2 & 2 & 1 & $x_{2} x_{3}$ \\
\hline \begin{tabular}{|l|l|}
1 & 3 \\
\end{tabular} & \begin{tabular}{|l|l|}
1 & 3 \\
\end{tabular} & \begin{tabular}{|l|l|}
0 & 1 \\
\end{tabular} & \\
\hline 3 & 3 & 2 & \\
\hline 2 & 2 & 1 & $x_{2} x_{3}^{2}$ \\
\hline 1 & 1 & 0 & \\
\hline
\end{tabular}

$$
i(S)=\begin{array}{|l|l|l|}
\hline 1 & 2 & \\
\hline 0 & 0 & 1 \\
\hline
\end{array}
$$

Now, using the tableaux $T$ and $i(S)$, cells of $T$ giving variable index and corresponding cell of $i(S)$ giving exponent, we build monomials $\mathbf{x}_{T}^{i(S)}$ as follow.

$$
\begin{aligned}
& T=\begin{array}{|l|l|l|l|l|l|l|}
\hline 2 & 4 & & 1 & 2 & \\
\hline 1 & 3 & 5
\end{array} \quad i(S)=\begin{array}{llll}
1 & 0 & 0 & 1 \\
\hline
\end{array} \\
& \mathbf{x}_{T}^{i(S)}=x_{1}^{0} x_{2}^{1} x_{3}^{0} x_{4}^{2} x_{5}^{1}
\end{aligned}
$$

Here are all the monomials in three variables.

For $T$ a standard tableaux of shape $\lambda$, let $R(T)$ and $C(T)$ denote the row stabilizer and the column stabilizer of $T$ respectively and consider the Young symmetrizer

$$
\epsilon_{T}:=\sum_{\sigma \in R(T)} \sum_{\tau \in C(T)} \operatorname{sign}(\tau) \tau \sigma
$$

which is an element of the group algebra $\mathbb{Q}\left[\mathfrak{S}_{n}\right]$. We know define the polynomial $F_{T}^{S}$ by

$$
F_{T}^{S}\left(x_{1}, \ldots, x_{n}\right):=\epsilon_{T}\left(\mathbf{x}_{T}^{i(S)}\right)
$$


Theorem 3.1. Let $n$ a positive integer, the family of $n$ ! polynomials $F_{T}^{S}$ for $S, T$ running over standard tableaux of the same shape form a basis of the $\operatorname{Sym}(\mathbf{x})$ module $\mathbb{K}[\mathbf{x}]$.

Terasoma and Yamada proved it using the usual bilinear form in its context : the divided difference associated to the longest element of the symmetric group [21.

In Three variable, here is the basis of $\mathbb{K}\left[x_{1}, x_{2}, x_{3}\right]$ as a $\operatorname{Sym}\left(x_{1}, x_{2}, x_{3}\right)$-module.

\begin{tabular}{|c|c|c|c|}
\hline$S$ & $T$ & $\mathbf{x}_{T}^{i(S)}$ & $F_{T}^{S}$ \\
\hline \begin{tabular}{|l|l|l|}
1 & 2 & 3 \\
\end{tabular} & \begin{tabular}{|l|l|l|}
1 & 2 & 3 \\
\end{tabular} & 1 & 6 \\
\hline \begin{tabular}{|l|l|}
3 & \\
1 & 2 \\
\end{tabular} & \begin{tabular}{|l|l|}
3 & \\
1 & 2 \\
\end{tabular} & $x_{3}$ & $2\left(x_{3}-x_{1}\right)$ \\
\hline \begin{tabular}{|l|l|}
3 & \\
1 & 2 \\
\end{tabular} & \begin{tabular}{|l|l|}
2 & \\
1 & 3 \\
\end{tabular} & $x_{2}$ & $2\left(x_{2}-x_{1}\right)$ \\
\hline \begin{tabular}{|l|l|}
2 & \\
1 & 3 \\
\end{tabular} & \begin{tabular}{|l|l|}
3 & \\
1 & 2 \\
\end{tabular} & $x_{2} x_{3}$ & $x_{2}\left(x_{3}-x_{1}\right)$ \\
\hline \begin{tabular}{|l|l|}
2 & \multicolumn{1}{|l}{} \\
1 & 3 \\
\end{tabular} & \begin{tabular}{|l|l|}
2 & \\
1 & 3 \\
\end{tabular} & $x_{2} x_{3}$ & $x_{3}\left(x_{2}-x_{1}\right)$ \\
\hline \begin{tabular}{|l|}
3 \\
2 \\
\end{tabular} & \begin{tabular}{|l|}
3 \\
2 \\
\end{tabular} & $x_{2} x_{3}^{2}$ & $\left(x_{3}-x_{1}\right)\left(x_{3}-x_{2}\right)\left(x_{2}-x_{1}\right)$ \\
\hline 1 & 1 & & \\
\hline
\end{tabular}

We will know try to solve Problem 2.2 by searching linear combination of higher Specht polynomials stabilized by the action of a permutation group.

\section{Combinatorial Description OF the invariant RiNG}

We now try to slice the invariant ring finer than degree by degree. As irreducible representations of the symmetric group are homogeneous, we will build format series mixing degree statistic and partitions.

4.1. A refinement of the Moliens series. Let $G \subset \mathfrak{S}_{n}$ a permutation group. Any module $\mathfrak{S}_{n}$-stable is also $G$-stable, thus any representation of $\mathfrak{S}_{n}$ is also a representation of $G$. Usually, An irreducible representation of $\mathfrak{S}_{n}$ will not stay irreducible when restricted to $G$. We are searching trivial representations of $G$ inside irreducible representation of $\mathfrak{S}_{n}$ and that can be done with a scalar product of character.

For $G$ a permutation group, we will denote $\mathcal{C}(G)$ the set of conjugacy classes of $G$. The usual scalar product between two characters $\chi$ and $\psi$ of $G$ is given by

$$
\langle\chi, \psi\rangle=\frac{1}{|G|} \sum_{C \in \mathcal{C}(G)}|C| \chi(\sigma) \psi(\sigma) \quad(\sigma \text { chosen arbitrary } \in C)
$$

Proposition 4.1. Let $\lambda \vdash n$ a partition of the positive integer $n$. Let $G \subset \mathfrak{S}_{n}$ a permutation group. The multiplicity of the trivial representation of $G$ inside the irreducible representation of $\mathfrak{S}_{n}$ indexed by $\lambda$ is given by $m_{\lambda}\left(G, \mathfrak{S}_{n}\right)$ with

(10) $m_{\lambda}\left(G, \mathfrak{S}_{n}\right):=\frac{1}{|G|} \sum_{C \in \mathcal{C}(G)}|C| M_{(\lambda, \text { cycle type }(\sigma))}, \quad(\sigma$ chosen arbitrary $\in C)$ 
where $M_{(\lambda, \text { cycletype }(\sigma))}$ is the coefficient of the character table of $\mathfrak{S}_{n}$ indexed by the partitions $\lambda$ and cycle type $(\sigma)$.

Proof. This just consist in using the usual scalar product of characters for $G$ with the trivial character of $G$. Thus we can remark that value of characters can be read on the character table of $\mathfrak{S}_{n}$ because conjugacy classes of $G$ are subset of conjugacy classes of $\mathfrak{S}_{n}$ and traces of matrices do not change when a representation of $\mathfrak{S}_{n}$ is viewed as a representation of $G$.

Definition 4.2. Let $G \subset \mathfrak{S}_{n}$ a permutation group. Using a formal set of variable $\mathbf{t}=\left(t_{\lambda}\right)_{\lambda \vdash n}$ indexed by partitions of $n$, we define the trivial multiplicities enumerator $P(G, \mathbf{t})$ as follow

$$
P(G, \mathbf{t}):=\sum_{\lambda \vdash n} m_{\lambda}\left(G, \mathfrak{S}_{n}\right) t_{\lambda}
$$

$P(G, \mathbf{t})$ count the multiplicities of the trivial representation of $G$ inside the irreducible representations of the symmetric group of degree $n$ themselves indexed by the partitions of the integer $n$.

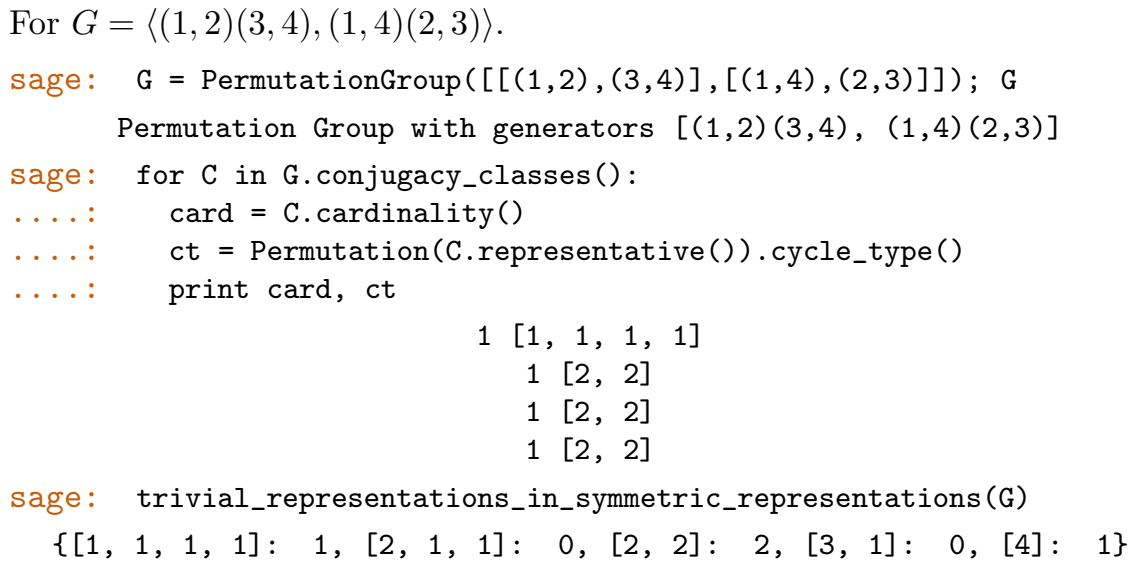

For this group, we thus have

$$
P(G, \mathbf{t})=t_{[1,1,1,1]}+2 t_{[2,2]}+t_{[4]}
$$

Definition 4.3. Let $\lambda \vdash n$ a partition of a positive integer $n$ and $z$ a formal variable. The will denote $\phi(\lambda, z)$ the representation appearance polynomial defined as follow

$$
\phi(\lambda, z):=\sum_{T \in S T a b(\lambda)} z^{\text {cocharge }(T)}
$$

Where the sum run over all standard tableaux $T$ of shape $\lambda$.

$\phi(\lambda, z)$ make the link between the degree $z$ and the irreducible representations of $\mathfrak{S}_{n}$ isomorphic the abstract one indexed by $\lambda$ appearing inside the quotient $\mathbb{K}[\mathbf{x}] / \operatorname{Sym}^{+}(\mathbf{x}) \cdot \phi(\lambda, 1)=|S T a b(\lambda)|$ give the multiplicities of the irreducible representation indexed by $\lambda$. Generally, if $\phi(\lambda, z)$ has coefficient an integer $k$ for a term in $z^{d}$, this means that $k \operatorname{Sym}(\mathbf{x})$-module isomorphic to the irreducible representation of $\mathfrak{S}_{n}$ indexed by $\lambda$ can be built inside the graded quotient $\mathbb{K}[\mathbf{x}] / \operatorname{Sym}^{+}(\mathbf{x})$ at degree $d$. The higher Specht Polynomials realize explicitly these representations 
because the cocharge is exactly the sum of the entries of tableau $i(S)$ (or the degree of the corresponding Specht).

Proposition 4.4. Let $G \subset \mathfrak{S}_{n}$ a permutation group. The trivial multiplicities enumerator $P(G, t)$ and the Hilbert series $H(G, z)$ are related by

$$
H(G, z)=\frac{P\left(G, t_{\lambda} \rightarrow \phi(\lambda, z)\right)}{(1-z)\left(1-z^{2}\right) \cdots\left(1-z^{n}\right)}
$$

Proof. This result is a consequence of some statements about the combinatorics of standard tableaux. As discussed previously, once we have the dimension of $G$ trivial space inside each irreducible representation, it remains to know at which degree copies of irreducible representation lies into the quotient $\mathbb{K}[\mathbf{x}] / \operatorname{Sym}^{+}(\mathbf{x})$. The cocharge of standard tableaux is the right statistic partitioning the occurrences of $\mathfrak{S}_{n}$-spaces along the degree.

Back with the example $G=\langle(1,2)(3,4),(1,4)(2,3)\rangle$,

$$
\begin{aligned}
& \text { sage: } \quad G=\text { PermutationGroup }([[(1,2),(3,4)],[(1,4),(2,3)]]) ; G \\
& \text { Permutation Group with generators }[(1,2)(3,4),(1,4)(2,3)] \\
& \text { sage: G.molien_series() } \\
& \left(x^{2}-x+1\right) /\left(x^{6}-2 x^{5}-x^{4}+4 x^{3}-x^{2}-2 x+1\right) \\
& \text { sage: } \quad \mathrm{S} 4=\text { SymmetricGroup (4); S4 } \\
& \text { Symmetric group of order } 4 ! \text { as a permutation group } \\
& \text { sage: S4.molien_series() } \\
& 1 /\left(x^{10}-x^{9}-x^{8}+2 x^{5}-x^{2}-x+1\right) \\
& \text { sage: G.molien_series() / S4.molien_series() } \\
& x^{6}+2 x^{4}+2 x^{2}+1
\end{aligned}
$$

On the other side, we had

$$
P(G, \mathbf{t})=t_{[1,1,1,1]}+2 t_{[2,2]}+t_{[4]}
$$

Let us now list standard tableaux of shape $[1,1,1,1],[2,2] \operatorname{and}[4]$.

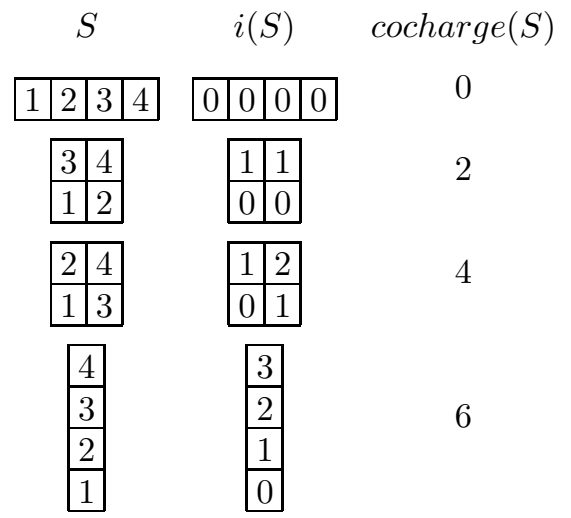

We have $\phi([1,1,1,1], z)=1, \phi([2,2], z)=z^{2}+z^{4}$ and $\phi([4], z)=z^{6}$. Injecting these evaluations inside the trivial multiplicities enumerator, we recover

$$
P\left(G, t_{\lambda} \rightarrow \phi(\lambda, z)\right)=1+2 z^{2}+2 z^{4}+z^{6}
$$


which give the number of secondary invariants degree by degree when elementary symmetric polynomials are taken as primary invariants. This polynomials is also the quotient of the two Hilbert series that can be computed in GAP [8] using Molien's Formula.

4.2. Secondary invariants built from higher Specht polynomial. Let $G \in$ $\mathfrak{S}_{n}$ a permutation group, $\lambda \vdash n$ a partition of $n$. Let us suppose that we have calculated $m_{\lambda}\left(G, \mathfrak{S}_{n}\right)$ and we now want to build explicitly the secondary invariant polynomials. We are in the case in which we have an homogeneous $G$-stable space inside which we want to construct a finite and known number of independent invariant polynomials under the action of $G$.

The usual way to dealt with this problem is to built an explicit family spanning the concerned space by generating polynomials forming a basis. Then basis element by basis element, we apply the Reynolds operator and some linear algebra to get a free family of the wanted dimension. knowing this dimension give a stopping criteria often very important since computations are extremely heavy even for small number of variables.

In our context, even the usual approach would work, as permutation are often given by a list of generators, we can even forget the Reynolds operator.

Proposition 4.5. Let $G \in \mathfrak{S}_{n}$ a permutation group given by some generator : $G=$ $\left\langle\sigma_{1}, \ldots, \sigma_{r}\right\rangle$. Let $\lambda \vdash n$ a partition. The $G$-trivial abstract space inside the abstract representation of $\mathfrak{S}_{n}$ indexed by $\lambda$ is given by the intersection of the eigenspace of the representation matrices of $\sigma_{1}, \ldots, \sigma_{r}$ associated the eigenvalue 1 .

Proof. We view here representation indexed by $\lambda$ as the formal free module generated by the standard tableaux of shape $\lambda$. We know that a subspace of dimension $m_{\lambda}\left(G, \mathfrak{S}_{n}\right)$ have its elements invariant under the action of $G$. Being invariant under $G$ is equivalent to be stabilized by the Reynolds operator but is also equivalent (by definition in fact) to be stabilized by the action of the generators of $G$. Since we are working inside a representation of $\mathfrak{S}_{n}$, each permutation have an associated matrix and the kernel of this matrix characterize the formal subspace stabilized

\section{Algorithm BUILDing SECONDARY INVARIANTS}

We now present an effective algorithm exploiting the approach using the representation of the symmetric group.

Computation dependencies :

- Character table of the symmetric group

- Conjugacy classes of the group $G$ (cardinalities and representatives)

- Matrices of irreducible representation of the symmetric group

- Some linear algebra

The returned set is composed by linear combinations of higher Specht polynomials. These polynomials can be easily evaluated but, as they contains a lot of Vandermonde factors, there expansion on a set of $n$ formal variables is an heavy computation. 


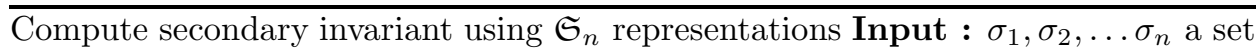
of permutations of size $n$ generating a group $G$.

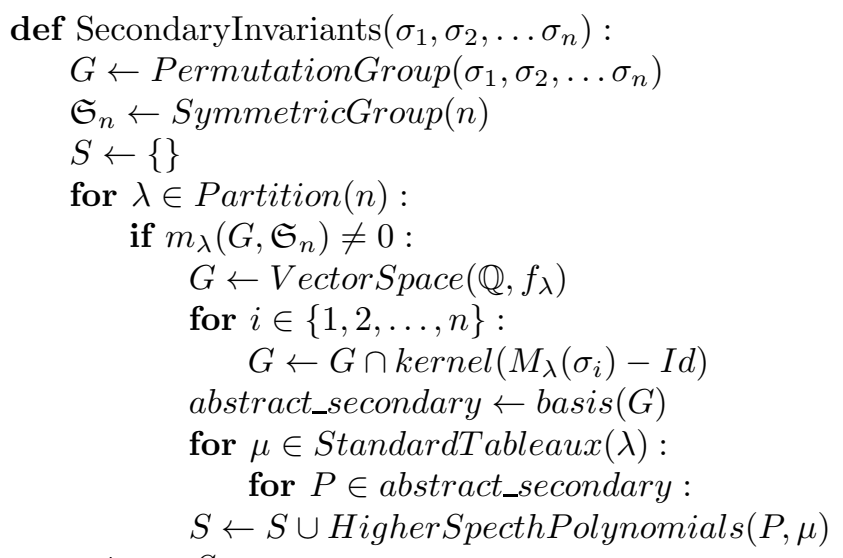

return $S$

5.1. A large trace of the algorithm. A teen years computational challenge consist in computing a generating family of the ring of invariants of the group acting on the edges of graphs over 5 nodes. This group is a subgroup of the symmetric group of degree $10=\left(\begin{array}{l}5 \\ 2\end{array}\right)$ and has for cardinality $5 !=120$. As far as we know, no computer algebra system has already handle a such computation. After 12 hours of computation, Magma, Singular and the evaluation approach written in Sage did not finish (in Sage, more precisely, around 5 percent of this computation(linear algebra) was done after 24 hours). We tried our approach on this group and we got the following verbose :

The trace have should be read has the following pattern

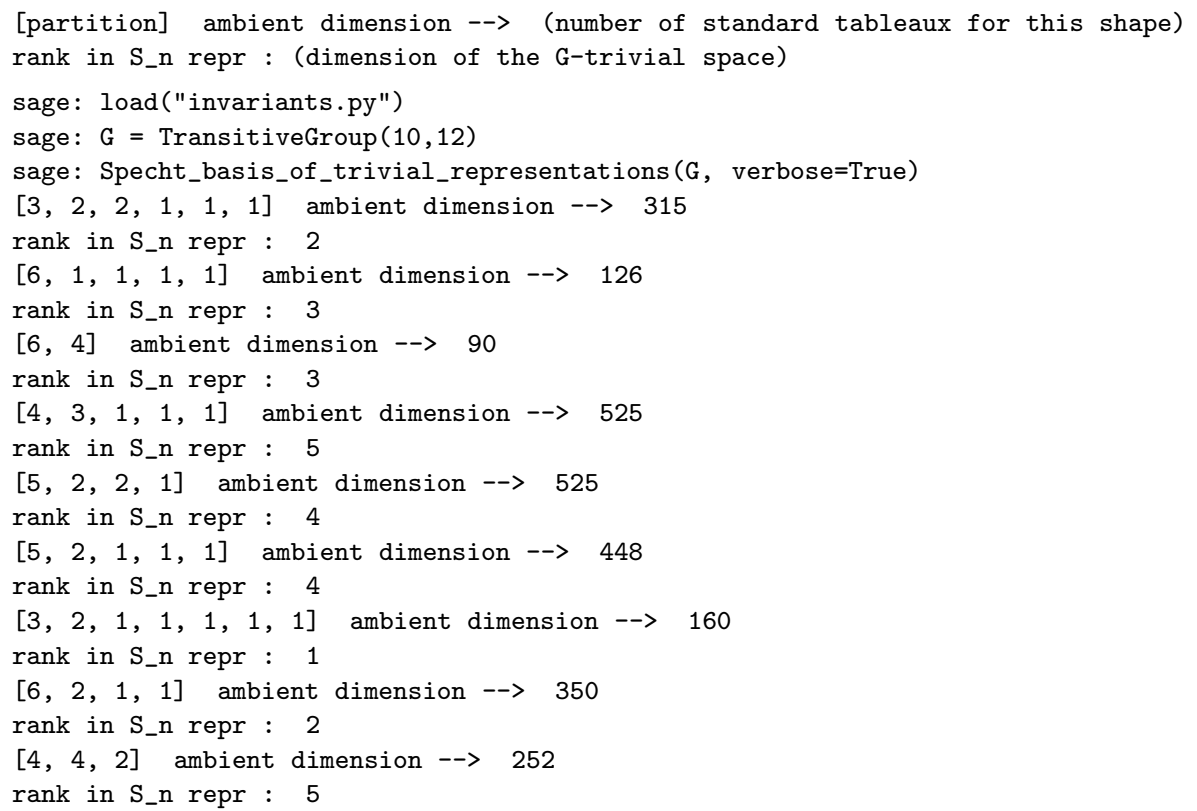




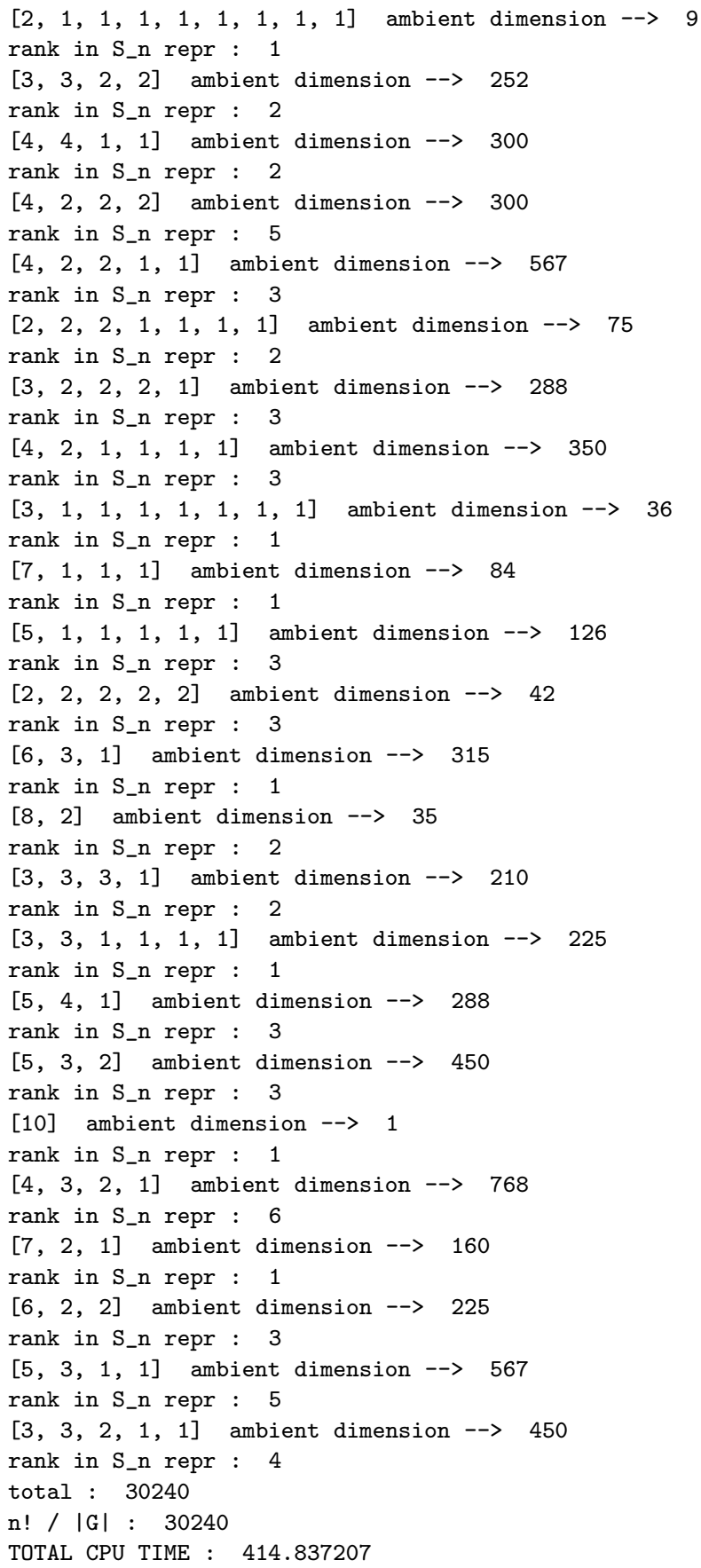

Our algorithm took 414 seconds to generated the 30240 secondary invariants as linear combinations of higher Specht polynomials. We still believe that the computation of a couple primary and secondary invariants for this group is unreachable for Magma, Singular and the evaluation approach in less than 24 hours. 
For example, inside the symmetric representation associated with the partition $(4,3,2,1)$. The algorithm making the Gauss reduction build a space of dimension 6 inside an ambient space of dimension 768. Using the Hook-length formula, on can check that there exist 768 standard tableaux of shape $(4,3,2,1)$

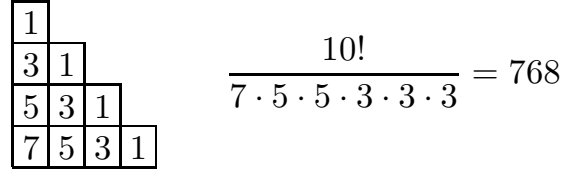

5.2. Implementation details. Our approach involves a lot a technology around representation theory and symbolic computation, most of the required prerequisites are available in current computer algebras system. For our problem, dependencies have been implemented in GAP [8] or Sage 19. We implemented a first version in Sage (400 lines of code with tests and documentation) to test the efficienty of this approach.

Here are the main steps of computation.

- Compute the character table of the symmetric group.

This task is done by GAP [8]. The Murnaghan-Nakayama rule is an example of working algorithm. This is a small part of computations and it can also be precomputed and store for all small symmetric group (for degree at most $20)$.

- Enumerate cardinality and a representative of each conjugacy class of $G$. Also handled by GAP 8 and interfaced in Sage 19. The general problem of enumerating conjugacy classes of finite group is not simple but there are efficient algorithms for permutation group.

- Compute the trivial multiplicities enumerator of $G$.

We computed a short function which just iterate over all partitions and compute a simple scalar product (see Formula 10).

- Calculate the matrices of $\sigma_{1}, \sigma_{2}, \ldots, \sigma_{n}$ inside the irreducible representations of $\mathfrak{S}_{n}$.

Once we have identified a $G$-trivial space inside an irreducible of $\mathfrak{S}_{n}$, we build the matrices of the generators of $G$ using the Sage [19] object : SymmetricGroupRepresentation which admits as argument a partition. The returned object is able to build matrices of the abstract representation from a permutation given in line notation.

- Compute the intersection of stabilized subspace of these matrices.

We use in a loop the method intersection of Sage VectorSpace and the method kernel of Sage Matrix class. Our linear algebra is thus handle by Sage [19].

- Transcript abstract combinations of standard tableaux in term of higher Specht polynomials.

Sage and especially Sage-Combinat 17. contains the combinatorial family of standard tableaux. A lot of combinatorial statistic are availlable on such objects like the cocharge. We implemented the higher Specht polynomials as Sage polynomials computed from a pair of Sage StandardTableaux of the same shape.

Our code is completely not optimized. Since most of computations in invariants (minimal generating set, secondary invariants, Hironaka decomposition) present 
large theoretical exponential complexity, we believe that, before code source refinement, we need new algorithms with better asymptotically behavior. However, a huge factor of execution time can easily be wined from our code with cache remember, better interface between GAP and Sage, parralelisation (each computer core can echelonize matrices for a special irreducible representation), we wanted to benchmarks our approach using the current available (and open source) technology. Therefore, anyone (even a student) can reproduce our experience rapidly and for free.

5.3. Complexity. The rich literature about effective invariant theory does not provide a lot a fin complexity bound for algorithms. Gröbner basis admit very general complexity bounds (in worst case $2^{2^{O(n)}}$ for $n$ variables) which appears to be overestimated compared to their effective behavior. The thesis [3] of the author present an evaluation approach to compute the invariants inside a quotient of a reduced dimension. The algorithm computing secondary with this technique has a complexity in $O\left((n !)^{2}+\frac{(n !)^{3}}{\left.G\right|^{2}}\right)$. Using the representation of the symmetric group, it is still very hard to establish a fin bound. However, we can produce better bounds.

Theorem 5.1. Let $G$ a permutation group, subgroup of $\mathfrak{S}_{n}$, given by $r$ generators. The complexity of the linear algebra reduction in algorithm computing the secondary invariants of $G$ in Section 5 is bounded by

$$
r \cdot\left(\sum_{\lambda \vdash n} m_{\lambda}\left(|G|, \mathfrak{S}_{n}\right)\left(f_{\lambda}\right)^{2}\right)
$$

where $f_{\lambda}$ is the number of standard tableaux of shape $\lambda$ and $p(n)$ is the number of partition of size $n$.

Proof. This is just a straightforward counting of the reductions the $r$ matrices of permutation for each irreducible representation of the symmetric group. For each partition $\lambda$ of $n$, we have to construct a free family of $m_{\lambda}\left(|G|, \mathfrak{S}_{n}\right)$ vectors (i.e. the rank) inside a space of dimension $f^{\lambda}$ (i.e. number of indeterminates) from $r \cdot f^{\lambda}$ equations (the vertical concatenation of the $r$ matrices). Summing these operations give the announced bound.

Corollary 5.2. Let $G$ a permutation group, subgroup of $\mathfrak{S}_{n}$, given by $r$ generators. The complexity of the algorithm computing the secondary invariants in Section 5 has a complexity in $O\left(r \cdot(n !)^{\frac{3}{2}}\right)$.

Proof. Let us denote $f_{\max }:=\max _{\lambda \vdash n}\left\{f^{\lambda}\right\}$. We thus have

$$
r \cdot\left(\sum_{\lambda \vdash n} m_{\lambda}\left(|G|, \mathfrak{S}_{n}\right)\left(f_{\lambda}\right)^{2}\right) \leqslant r \cdot\left(\sum_{\lambda \vdash n} f_{\max }\left(f_{\lambda}\right)^{2}\right)
$$

since, at worse, the irreducible representation of $\mathfrak{S}_{n}$ is composed only by element stabilized point wise by $G$. The $f_{\max }$ term can go out the sum and using formula 6 we get the bound

$$
r \cdot f_{\max } \cdot n !
$$

Using the triangular inequality, we roughly have $f_{\max }^{2} \leqslant n$ ! and that give the result. 


\section{FurThER DEVELOPMENTS}

Even the impressive algorithmic efficiently of this approach using the representation of the symmetric group, it has also the advantage of putting a lot of combinatrics inside this problem often classed inside effective algebraic geometry.

\section{ACKNOWLEDGEMENTS}

This research was driven by computer exploration using the open-source mathematical software Sage [19]. In particular, we perused its algebraic combinatorics features developed by the Sage-Combinat community [17, as well as its group theoretical features provided by GAP 8 .

\section{REFERENCES}

[1] I. Abdeljaouad. Théorie des Invariants et Applications à la Théorie de Galois effective. PhD thesis, Université Paris 6, 2000.

[2] F. Bergeron. Algebraic combinatorics and coinvariant spaces. CMS Treatises in Mathematics. 2009.

[3] N. Borie. Calcul des invariants des groupes de permutations par transformée de Fourier. $\mathrm{PhD}$ thesis, Laboratoire de Mathématiques, Université Paris Sud, 2011.

[4] A. Colin. Solving a system of algebraic equations with symmetries. J. Pure Appl. Algebra, 117/118:195-215, 1997. Algorithms for algebra (Eindhoven, 1996).

[5] A. Colin. Théorie des invariants effective; Applications à la théorie de Galois et à la résolution de systèmes algébriques; Implantation en AXIOM. PhD thesis, École polytechnique, 1997.

[6] H. Derksen and G. Kemper. Computational invariant theory. Springer-Verlag, Berlin, 2002.

[7] J. Faugère and S. Rahmany. Solving systems of polynomial equations with symmetries using SAGBI-Gröbner bases. In Proceedings of the 2009 international symposium on Symbolic and algebraic computation, pages 151-158, 2009.

[8] The GAP Group, Lehrstuhl D für Mathematik, RWTH Aachen, Germany and SMCS, U. St. Andrews, Scotland. GAP - Groups, Algorithms, and Programming, 1997.

[9] A. Garsia and D. Stanton. Group actions on stanley-reisner rings and invariants of permutation groups. Advances in Mathematics, 51(2):107 - 201, 1984.

[10] K. Gatermann. Symbolic solution of polynomial equation systems with symmetry. KonradZuse-Zentrum für Informationstechnik Berlin, 1990.

[11] K. Geissler and J. Klüners. Galois group computation for rational polynomials. J. Symbolic Comput., 30(6):653-674, 2000. Algorithmic methods in Galois theory.

[12] G. Kemper. The invar package for calculating rings of invariants. IWR Preprint 93-94, University of Heidelberg, 1993.

[13] S. King. Fast Computation of Secondary Invariants. Arxiv preprint math/0701270, 2007.

[14] S. King. Minimal generating sets of non-modular invariant rings of finite groups. Arxiv preprint math/0703035, 2007.

[15] A. Lascoux and M.-P. Schützenberger. Polynômes de Schubert. C. R. Acad. Sci. Paris Sér. I Math., 294(13):447-450, 1982.

[16] M. Pouzet and N. M. Thiéry. Invariants algébriques de graphes et reconstruction. C. R. Acad. Sci. Paris Sér. I Math., 333(9):821-826, 2001.

[17] T. Sage-Combinat community. Sage-Combinat: enhancing Sage as a toolbox for computer exploration in algebraic combinatorics, 2008.

[18] R. P. Stanley. Invariants of finite groups and their applications to combinatorics. Bull. Amer. Math. Soc. (N.S.), 1(3):475-511, 1979.

[19] W. Stein et al. Sage Mathematics Software (Version 3.3). The Sage Development Team, 2009. http://www. sagemath.org.

[20] B. Sturmfels. Algorithms in invariant theory. Springer-Verlag, Vienna, 1993.

[21] T. Terasoma and H. Yamada. Higher Specht polynomials for the symmetric group. Proc. Japan Acad. Ser. A Math. Sci., 69(2):41-44, 1993.

[22] N. M. Thiéry. Algebraic invariants of graphs: a study based on computer exploration. SIGSAM Bulletin, 34(3):9-20, Sept. 2000. 
[23] N. M. Thiéry. Computing minimal generating sets of invariant rings of permutation groups with SAGBI-Gröbner basis. In Discrete models (Paris, 2001), pages 315-328 (electronic). 2001.

Univ. Paris Est Marne-La-Vallée, Laboratoire d'Informatique Gaspard Monge, Cité Descartes, Bât Copernic - 5, bD Descartes Champs sur Marne 77454 Marne-la-Vallée Cedex 2, France 